Research Article

\title{
The Contribution of Psychotherapists to Patient Care in Multidisciplinary Heart Transplantation Care Teams
}

Katharina Tigges-Limmer ${ }^{1,{ }^{+},{ }^{*} \text {, Stephan Ensminger }}{ }^{2,{ }^{\dagger}}$, Yvonne Brocks ${ }^{1,{ }^{\dagger}}$, Scott Stock Gissendanner ${ }^{3,+}$, Jan Gummert ${ }^{1,+}$

1. Department of Thoracic and Cardiovascular Surgery, Heart and Diabetes Center NRW, RuhrUniversity Bochum, Bad Oeynhausen, Germany; E-Mails: ktigges-limmer@hdz-nrw.de; ybrocks@hdz-nrw.de; jgummert@hdz-nrw.de

2. Department of Cardiac and Thoracic Vascular Surgery, University Heart Center Lübeck, Germany; E-Mail: stephan.ensminger@uksh.de;

3. Social Science Faculty, Georg August University, Göttingen, Germany; E-Mail: sgissen@gwdg.de

+ These authors contributed equally to this work.

* Correspondence: Katharina Tigges-Limmer; E-Mail: ktigges-limmer@hdz-nrw.de

Academic Editor: Yasuhiko Sugawara

Special Issue: Perspectives on Heart Transplantation

OBM Transplantation

2019, volume 3 , issue 1

doi:10.21926/obm.transplant.1901056
Received: October 28, 2018

Accepted: February 14, 2019

Published: March 05, 2019

\begin{abstract}
Background: The participation of mental health professionals in multidisciplinary care teams for heart transplantation has become an international standard practice. The contribution of these professionals in this setting, which involves assessment and interventions, has not been the subject of systematic study.

Methods: This paper explores how psychotherapists contribute to multidisciplinary decision making and care in transplant teams before, during, and after transplantation. To illustrate, it uses the case of a 19-year-old woman with anorexia nervosa who underwent heart transplant at a large transplantation center in Germany. She received psychotherapeutic interventions intended to lower the risk of non-adherence.
\end{abstract}

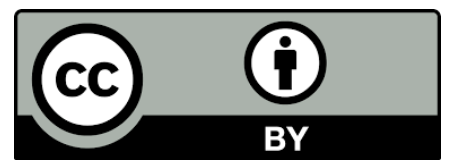

(C) 2019 by the author. This is an open access article distributed under the conditions of the Creative Commons by Attribution License, which permits unrestricted use, distribution, and reproduction in any medium or format, provided the original work is correctly cited. 
Results: This first reported case of heart transplant in a patient with a previous diagnosis of anorexia nervosa also documents psychotherapeutic interventions that were favorable for sustainable weight-gain and how the multidisciplinary environment enabled their development and implementation. We provide details on the contribution of the psychologist in the case, including hypnotherapy interventions and adjustments in routine nursing practices.

Conclusions: This paper shows how mental health professionals working in multidisciplinary teams improve patient outcomes in organ transplantation. In order to collect and systematize clinical knowledge of psychotherapeutic interventions for transplant candidates with mental health issues, additional studies with multiple centers, multiple cases, and a range of mental health disorders are needed.

\section{Keywords}

Heart transplant; anorexia nervosa; mental health; multidisciplinary care; psychotherapy; intervention; case report

\section{Introduction}

Orthotopic heart transplantation following the guidelines of the International Society of Heart and Lung Transplantation (ISHLT) is conducted by multidisciplinary care teams whose members include physicians, surgeons, nurses, social workers, immunologists, pharmacists, dieticians, and other specialists as needed [1, 2]. The provision of continuous support by mental health professionals working on these teams has been recognized as good practice for years [3], is recommended in ISHLT guidelines [1, 4], is instituted in the bylaws of the US Organ Procurement and Transplantation Network [5], and has been most recently incorporated into German transplantation law [6].

Depending on national and clinical settings, mental health professionals in multidisciplinary heart transplantation teams may be psychiatrists, psychotherapists, specially trained nurses, or social workers. They have four main areas of responsibility: diagnosing psychiatric disorders, assessing psychosocial resources and risks, providing psychotherapeutic interventions, and educating patients with the goal of reducing health risks and activating resources for better life quality and coping [7]. Typical therapies are short psychotherapeutic interventions that comprise elements of supportive therapy, resource activation and future orientation, social support, dissociation and association of other burdens, cognitive restructuring, and relaxation and painrelieving techniques [8]. The number of consultations each patient receives can vary widely among transplant centers and patients. One of the rare empirical studies reported an average of 2.2 consultations before surgery and 3.0 afterwards but a total of 16.6 if the patient first received a mechanical circulatory assist device [9]. In our transplant center, 25 or more consultations per patient is not unusual.

The most common comorbid psychological problems among heart transplant patients are major depressive episodes, adjustment disorders, and surgery-related post-traumatic stress reactions $[8,10]$. Patients also may face psychosocial challenges to life-long adherence: 
psychopathology both precedent and concomitant to the onset of terminal heart disease, substance abuse and other risky habits, cognitive dysfunction, or the lack of social support [11, 12]. Common stressors are feelings of loss of control, fear of death, disruption of family roles and worrisome fantasies about acquiring character traits or memories from the heart donor. However, patients' disorders and distress are usually reliably manageable with standard psychotherapeutic interventions if the patient has adequate psychosocial resources and if the interventions are adequately modified in consideration of patients' poor cardiac health and reduced cognitive functioning immediately after surgery $[8,13]$.

The interaction of mental health professionals with other members of the care team is structured by several critical decisions. The first point of contact with the patient is often made by the mental health expert during the initial psychosocial screening and assessment, the components of which are outlined in Table 1. The patient's situation will then be discussed in an interdisciplinary transplantation conference in which all cases up for placement on the transplantation list are reviewed and decided upon. The team must decide whether the patient has an adequate chance of being successfully transplanted at the center in question and, then, whether and at what point the patient should be given high urgency (HU) priority on the donorheart waiting list. These decisions are informed by medical and psychosocial assessments of the patient's likelihood of surviving the surgical procedure and of being willing and able to adhere to a life-long pharmaceutical, dietary, and lifestyle regimen to minimize the risk of tissue rejection. The decision to approve transplantation despite a mental health contraindication hinges on the mental health professional's confidence that the patient will be able to control either the severity of psychological symptoms or their negative impact on adherence and other health related behaviors.

Table 1 Components of initial psychosocial assessment for heart transplant patients.

- Screening and diagnoses for previous and current psychiatric illnesses

- Cognitive testing

- Assessment of drug use and addiction, including nicotine and alcohol

- Assessment of adherence history, especially related to medication and medical advice

- Assessment of psychosocial barriers that could negatively affect patient health outcomes

- Assessment of available social support and the psychosocial burden on patient caregivers

- Financial risk assessment

Source: [4]

Following the initial decision to accept a candidate for transplantation, a treatment plan is created by the care team with the goal of improving the patient's overall physical and mental health in preparation for transplant. During this time, the physical condition of the patient is monitored to detect any deterioration that would justify HU status, if not already given. When a donor heart becomes available, the decision must be made then whether to prepare the patient and initiate surgery. After surgery, the team monitors patient health and adjusts treatment plans as needed when the patient is transferred out of intensive care. Periodic monitoring continues after discharge. 


\section{Materials and Methods}

In 2011, a patient with a previous diagnosis of anorexia nervosa underwent a successful transplant procedure at our transplant center and continues to be in good health today. We present her case below following the CARE guidelines for reporting clinical cases [14]. Information is provided regarding the patient's heart-disease parameters, the symptomatic expression of her eating disorder, and her psychosocial resources and burdens. We describe how this information was processed and acted upon by the multidisciplinary transplant team to decide on the treatment regimen prescribed for each stage of treatment and what actions the psychotherapist took during the course of treatment. This case illuminates the contribution of mental health professionals in multidisciplinary care teams through an account of the process by which one team had to make an unusually difficult decision, ultimately agreeing on the acceptance for transplantation of the patient, based in part on the assessment and anticipated support of the team's psychotherapist.

There are no reported cases of solid organ transplant involving a patient with a previous diagnosis of anorexia nervosa, although there are reports of anorexia symptoms developing after renal transplant [15]. The contribution of mental health specialists to decision making in solid organ transplant teams has been a subject of investigation only rarely [16-18].

The patient whose case is examined in the study participated in the decision to document her case history. She has given permission for publication.

\section{Case Report}

\subsection{Clinical Findings Prior to the Initial Interview}

The 19-year old female patient approached our center for a heart-transplant screening in 2011 at the age of 19 . The patient was diagnosed with dilated cardiomyopathy in early childhood and suffered consecutive symptoms of heart failure. In 2000, at the age of eight, she was treated for high grade mitral regurgitation with a mechanical mitral valve replacement (St. Jude-Medical 33 $\mathrm{mm}$ ). Physical exercise was limited from early childhood onward, but she reported that her first heart surgery had no negative psychological repercussions.

The patient had been previously diagnosed with anorexia nervosa, an eating disorder that results in severe undernourishment. At the age of 15, with a body mass index (BMI) of 14, the patient underwent compulsory psychiatric treatment and forced feeding. This was followed by a total of three inpatient and three outpatient treatments. Treatment was unsuccessful.

Anorexia nervosa is a contradiction for heart transplantation because undernourishment and underweight are independent predictors affecting survival after heart transplant [19, 20]. Although guidelines for the use of circulatory assist devices recommend weight gain before surgery for undernourished patients [21], there are no current evidence-based guidelines for evaluating the risk posed by eating disorders for heart transplantation, nor are eating disorders mentioned in the ISHLT transplant listing criteria. This stands in contrast to the recommendation to handle morbid obesity, defined as BMI of greater than $35 \mathrm{~kg} / \mathrm{m}^{2}$, as a contraindication and to initiate "weight loss to achieve a BMI of $<30 \mathrm{~kg} / \mathrm{m}^{2}$ or percent BMI of $<140 \%$ of target before listing for cardiac transplantation" [22]. For patients with a history of anorexia, recovery after transplantation is threatened not only by underweight and malnutrition but also by potential 
future interference with immunosuppressant intake. Autoaggressive starving, self-induced vomiting, excessive exercise, and body neglect can be detrimental. Moreover, the likely success of weight-gain interventions to achieve target $\mathrm{BMI}$ prior to transplant is reduced. Finally, due to the paucity of research on this unusual patient group, it is unclear what psychotherapeutic interventions can be effective in preventing a recurrence of symptoms and thus improving mortality risk and justifying transplant.

Early in 2011, the patient's cardiorespiratory failure progressed significantly and her cardiologist recommended heart transplantation. When the transplant center closest to her home refused treatment because of the anorexia, she came to our hospital seeking a second opinion. The anorexia diagnosis gave sufficient reason for denying consideration for orthotopic heart transplant, but refusing to treat would have meant almost certain death for the young patient.

\subsection{Diagnostics and Psychosocial Assessment}

In the psychosocial assessment, the patient presented herself as motivated and open for psychological reassessment and treatment in an active search for help. She reported always having been thin and having starved herself systematically since the age of 13 but that she rarely vomited intentionally, never induced diarrhea, and never took appetite suppressants. She recounted often feeling a desire to disappear into "thin" air. Given the many situations in which she felt "powerless," the crucial factor to her condition was apparently the feeling of power she obtained by starving herself. She noted always having had a lively temperament, but that her heart disease prevented her from being as active as she wanted. Consequently, physical activities were a source of contention with her mother beginning in childhood. Her mother was apt to violate boundaries, and the patient learned at an early age to provoke her by quarrelling. Starving herself was an additional provocation.

In her own opinion, previous psychotherapeutic interventions for her anorexia all failed due to her defiance. She had never been able to develop a trusting therapeutic relationship with any of the many psychotherapists she saw. Her symptoms improved when she moved into her own apartment. Then she was able to eat autonomously without being monitored or pressured and to achieve the then current BMI of 17 , albeit without ever menstruating. She adhered to her cardiac medication schedule without any problems, almost compulsively in fact. Thus, she demonstrated a capacity for independently securing her own survival by adhering to doctor's instructions and keeping her BMI above a low limit. However, she had never experienced successful treatment of the psychological aspects of her anorexia: perceptual disorders, negative and self-disparaging cognitions, and the inability to relieve stress.

The patient's psychological situation at the time of assessment involved rejection of her femininity, starvation as a means of control, a conflict-ridden family with delayed detachment, an entrenched psychiatric disorder, numerous subjectively failed therapy attempts, suspected problems with self-worth and compulsive reactance/defiance. Arguments with her mother frequently ended in self-induced vomiting. She articulated fears typical for patients facing heart transplantation, centering on death, complications during surgery, graft failure, and tissue rejection.

In sum, during the initial presentation the patient presented as alert, articulate, fully oriented, slightly tense, and cautious. Initially reticent, with time she became increasingly candid with the 
psychotherapist. The patient possessed psychological resources for maintaining a strict medical regimen including the categorical wish to live, goals, hobbies, and a caring attachment to her sister. Although the maternal relationship was conflict-ridden, it was not without loving moments. Psychotic symptoms or suicidal tendencies were not evident.

\subsection{Team Meetings and Therapeutic Interventions}

A multidisciplinary conference was initiated to decide whether to list the patient for transplantation. The team felt that the general prognosis regarding the anorexia was not good due to her previous resistance to psychotherapeutic treatment. The path to psychological recovery was deemed to be risky, as the pathology involved a complex set of factors including the patient's rejection of her femininity, starvation as a means of power and control, a family of origin experienced as conflict-ridden with delayed detachment, a deeply entrenched psychiatric disorder, numerous subjectively failed attempts at therapy, suspected problems with self-worth, as well as rapid reactance/defiance. Nonetheless, there was hope that her appetite could be stimulated by cortisone, which is routinely prescribed as part of immunosuppression, and optimism because the patient possessed valuable psychosocial resources including a credible will to live. Also, the risk that vomiting or provoked laxation would prevent immunosuppressive intake was estimated to be comparatively small in this case, due to the limited expression of anorexia symptoms.

After numerous discussions in 2011, the multidisciplinary care team decided to accept the patient and place her on the transplant waiting list. The entire team, especially the surgeons, wanted to do everything possible to give the young patient a chance to extend her life. Afterwards, the team met weekly for assessing and adjusting her therapies while awaiting transplant. Shortly thereafter, her cardiopulmonary health began to deteriorate and she was registered with Eurotransplant $^{\circledR}$ (Leiden, Netherlands) as "high urgency" for orthotopic heart transplantation. During the HU-waiting period, treatment continued to be monitored and adjusted in frequent team meetings.

The main psychotherapeutic goal set for the patient while waiting for transplantation surgery was to ready herself to integrate a new heart responsibly and sustainably in her pathologically problematic body schema and to promote compliance. The patient's history of never having established a trusting relationship with a psychotherapist after multiple psychotherapeutic interventions caused great concern initially for the assessing psychotherapist, who faced the burden of potentially being the only person in a position to help. Therefore, in the first therapeutic session after the initial psychosocial assessment, the patient's autonomy was explicitly underscored and her adulthood emphasized. In addition, a conscious distance was maintained to the starvation symptom with the message: "You alone are responsible for gaining enough weight for the heart transplant. That doesn't really interest me. What interests me is how you intend to integrate your new heart lovingly and kindly in your wonderfully obstinate body."

On the recommendation of the team's psychotherapist, the team decided to reinforce this psychotherapeutic intervention by allowing the patient to self-regulate her eating. Anorexia was treated as a non-issue. We expressly waived the requirement that the patient undergo dietary consultation and monitoring because she demonstrated a superb knowledge of nutrition and she had always vehemently rejected all forms of mandatory weight monitoring. She was instead given the task of autonomously maintaining her weight in the understanding that she would be unfit for 
transplantation if she could not meet a mutually agreed-upon target. It was hoped that this strategy would strengthen the patient's feeling of adult autonomy. The psychotherapist saw here an important opportunity for her and the team to demonstrate confidence in the patient's ability to hold a BMI of 17 and keep herself qualified for transplant. Importantly, this path was in keeping with professional ethics because the psychotherapist's primary mandate in this case was not to treat the anorexia but rather to help the patient integrate the donor heart.

Because cardiac insufficiency is commonly linked to emaciation, the ward's care team often treat underweight patients. This was, however, their first patient with anorexia. The nursing team accepted the idea of respecting the patient's dietary autonomy and implemented it conscientiously. The patient requested that weighing be done by the nursing staff without informing her of her exact weight because any mention of numbers triggered anorectically controlling, self-disparaging thoughts. It was astonishing to the team and a significant achievement for the patient that she managed to maintain her baseline weight during the threemonth HU waiting period without weighing herself. The patient reported post-operatively that she applied some of the advice given to her during her previous period of compulsory treatment. The freedom she was given to self-regulate her eating behavior allowed her to choose which methods to apply, a form of autonomy-reinforcement.

The second psychotherapeutic intervention was an analysis of the mother-daughter interaction. The patient had experienced her mother as overly intrusive. A pathological interaction between patient and mother, characterized by a struggle for autonomy with a simultaneous detachment delay, became apparent. Because the mother often visited, there was an initial thought to invite her into therapy to develop new patterns of communication in an attempt to set clear limits. However, the patient did not want to share the rapport she had built with the psychotherapist. This exercise of free will and self-assertion was taken as a positive sign. An appropriation almost akin to jealousy became clear.

The third intervention, also conducted during the waiting period, targeted the patient's low self-esteem, specifically her struggle to accept herself as valuable enough to receive a new heart. She described being unable to give herself enough space for self-care, of wanting to take up no space at all. Comments about her somatic-medical situation regularly led to self-deprecating cognitions and borderline depressive moods. The patient had intense doubts whether she was truly worthy of the gift of a new heart. She reported seldom receiving approbation or, if offered, being unable to accept it. The worry of being capable of truly accepting a new heart became a major topic for her shortly before transplantation, advancing to become her most fundamental, existential question. The interpretation of having been "thin air" more or less all her life, of being unable to give herself enough space for self-care and her own needs, of not wanting to take up space, all of which was expressed in the anorexia, clearly emerged. Premonitions of death and an occupation with mortal fears were significant at this juncture, and sometimes a yearning for death was articulated. For addressing these issues, an intervention based on two hypnotherapeutic mechanisms was conducted. The first mechanism, known in German hypnotherapy as the "therapeutic tertium" [23], refers to the construction of an interpersonal, imaginary configuration by the patient and therapist. The patient is encouraged to transfer onto it those internal strengths that the therapist helps her discover, thus personifying them into an internal champion for effecting change. In this way, when destructive cognitions undermine a patient's feeling of intrinsic self-worth, the creative unconscious can be strengthened and instrumentalized for 
healing and helping as a mediating authority between patient and psychotherapist. The therapeutic tertium is related to the psychotherapy concepts of shifting the "locus of control" and of projection, and it is also useful as practical means of helping patients grasp the mysterious and elusive aspects of their own psychology. The second mechanism, utilization [24], is a central idea of Eriksonian hypnotherapy and encompasses the idea that the therapist should utilize all of the patient's symptoms, character traits, and quirks for identifying and activating resources for reaching therapeutic goals. In the case at hand, the therapist and the patient visualized the patient's unconscious self as the therapeutic tertium, utilizing the patient's desire for autonomy and a low body weight as signs of mental strength. As the patient's trust in the ability of her body to assert itself against the intrinsic destructive anorectic cognitions (instrumentalized and dubbed the "anorexia witch") continuously grew, her confidence in the ability of her body to integrate a new heart also grew. This explanatory model proved effective for coping with anxiety.

Hypnotherapy involves inducing a trance-like state to lower dysfunctional defensive mechanisms and suggest positive changes. Patients can also learn to induce trances themselves, which is useful for controlling pain, fear, or stress. In this case, during trance induction the insubordination rule, i.e. the instruction that the patient may terminate the hypnosis session at any point [25], was clearly established out of consideration of the patient's heightened need for autonomy. Moreover, trance words like "heavy" were avoided in order to prevent reactance.

A final intervention sought to change the patient's perspective regarding her anorexia. The therapist helped the patient identify the root causes of her symptoms and find a way to distance herself from those symptoms without vilifying them. This process involved both hypnotherapy and cognitive behavioral therapy.

When a donor heart became available, there was no need for further deliberation regarding the patient's readiness. She was calm going into surgery. Immediately after the heart transplant, in intensive care, a hypnotherapeutic intervention was performed for a careful, loving integration of the organ [26]. The very emotional patient was able to feel the pulse of the donor heart, to welcome the new heart at length, to form an emotional bond to it and thus to initiate the process of embodiment-"from beat to beat, the foreign heart gradually becomes my own." As a posthypnotic suggestion, the patient was given the task of placing both hands on her heart whenever it felt comfortable to do so, and thus to build up a positive contact to the new heart in her own personal way. Here it became clear that the patient was overwhelmed by a need to say thank you, to welcome the new heart, and to introduce herself to it. For her, the heart transplant represented a chance to start a new chapter of self-care, self-acceptance, and reduced selfdeprecation.

The patient was prescribed a routine immunosuppressive regimen including initially $20 \mathrm{mg}$ of cortisone daily. No psychotropic medications were prescribed at any time.

\subsection{Follow-Up and Outcomes}

The post-operative course was normal, and the physically and mentally stable patient was discharged to her parents' home. However, during her stay in the conflict-ridden family situation, she experienced a recurrence of anorexia. The patient dialed an emergency hotline to the team psychotherapist and agreed to an outpatient crisis intervention in our clinic. She continued outpatient psychotherapy with another therapist and admitted herself on weekends to a 
psychosomatic clinic specialized in weight gain. This was the first time she had ever voluntarily agreed to outpatient psychotherapy. Her situation was stabilized, and as soon as the patient left her parents' home and returned to school, her situation normalized fully. The subsequent period was uneventful except for one episode in which the patient binged alcohol for the first time, resulting in a hospital overnight, and a mild, grief-triggered depressive episode three years after transplant. She also acted on her strong desire for a tattoo, which was extremely ill-advised medically. Both risky-behavior episodes can be interpreted as catching up with adolescent processes that previously had been denied to her by the cardiac insufficiency and the anorexia. For her tattoo, the patient chose a musical symbol on her hip, an erotic signal that could be interpreted as a step towards acceptance of her femininity. In the following year, she continually gained weight and reached a BMI of 22, the first time she had ever been within the normal range. During outpatient contact two years after heart transplantation, she reported having menstruated in the past four months and that she successfully graduated from school and was accepted to a university philosophy program. She also reported feeling no restrictions on daily activities or exercise. Sub-depressive setbacks (once or twice a month) remained difficult, but she countered these by writing poetry. She has required no further psychotherapy. The patient takes her immunosuppressive medication reliably and describes her relationship to her heart as loving. Twelve months after surgery there was one moderate rejection episode assessed as grade " $1 R^{\prime}$ " on the standard cardiac biopsy grading scale for cellular rejection [27], triggered by an infection which was successfully treated. Otherwise, the somatic course continues to be uneventful. Her current mental and somatic state, eight years after transplant, is stable.

Table 2 provides an overview of all psychotherapeutic interventions. A timeline of the case appears in Figure 1.

\section{Discussion}

This was the first case of heart transplantation in a patient with chronic anorexia and dilated cardiomyopathy at our transplant center. The treatment course to date has been successful. It involved many psychological treatment hours, frequent post-operative follow-ups initially, and one crisis intervention.

The transplantation care team in this case faced a situation similar to that described in Collins et al.'s pioneering article on the ethical problems that can arise in the psychological assessment of transplant patients with cognitive impairment or mental health issues, when decision making "can often hinge on information provided by the psychological consultant who is attempting to simultaneously serve the needs of the patient as well as the transplant team" [29]. In the case described here, the care team avoided this dilemma because the psychological problem at hand, anorexia, was amendable to psychotherapeutic interventions that reduced the risk of organ rejection. The psychotherapist worked with the patient and with the medical staff to find a feasible treatment path. 
Table 2 Psychotherapeutic interventions.

\begin{tabular}{ll} 
Intervention & $\begin{array}{l}\text { Frequency and } \\
\text { period of } \\
\text { consultations }\end{array}$ \\
\hline
\end{tabular}

Family systems therapy: Self- 2 in 1 week regulation and autonomy as a method to maintain BMI 17

Family systems therapy: Analysis of the mother relationship

Hypnotherapy: Reinforcing self-worth behavioral therapy: Change of perspective, understanding root cause of symptoms, distance without vilification of systems

Hypnotherapy: Welcoming of the new heart after surgery 1
13 in 5 weeks

4 in 2 weeks
Hypnotherapy and cognitive

\section{Patient's hospital room}

Patient's hospital room

Patient's hospital room

\section{Emotional relief,} discovery of the original context of the anorexia and denial of femininity

Strengthening personal efficacy and selfcontrol, emotional acceptance of self, therapist as model

Patient's hospital room

Widening the horizon, utilization of the unconscious as a therapeutic tertium, cognitive restructuring Intensive care unit after Integration of the waking from anesthesia
2 in 1 week
Crisis intervention
2 in 1 week
Therapist's
Regaining control over
office by anorexia symptoms
telephone and
in person Building adequate stress coping strategies
Cognitive behavioral therapy:
Outpatient Learning techniques of psychiatric clinic stress coping and for weight gain emotional regulation for anorexia control 


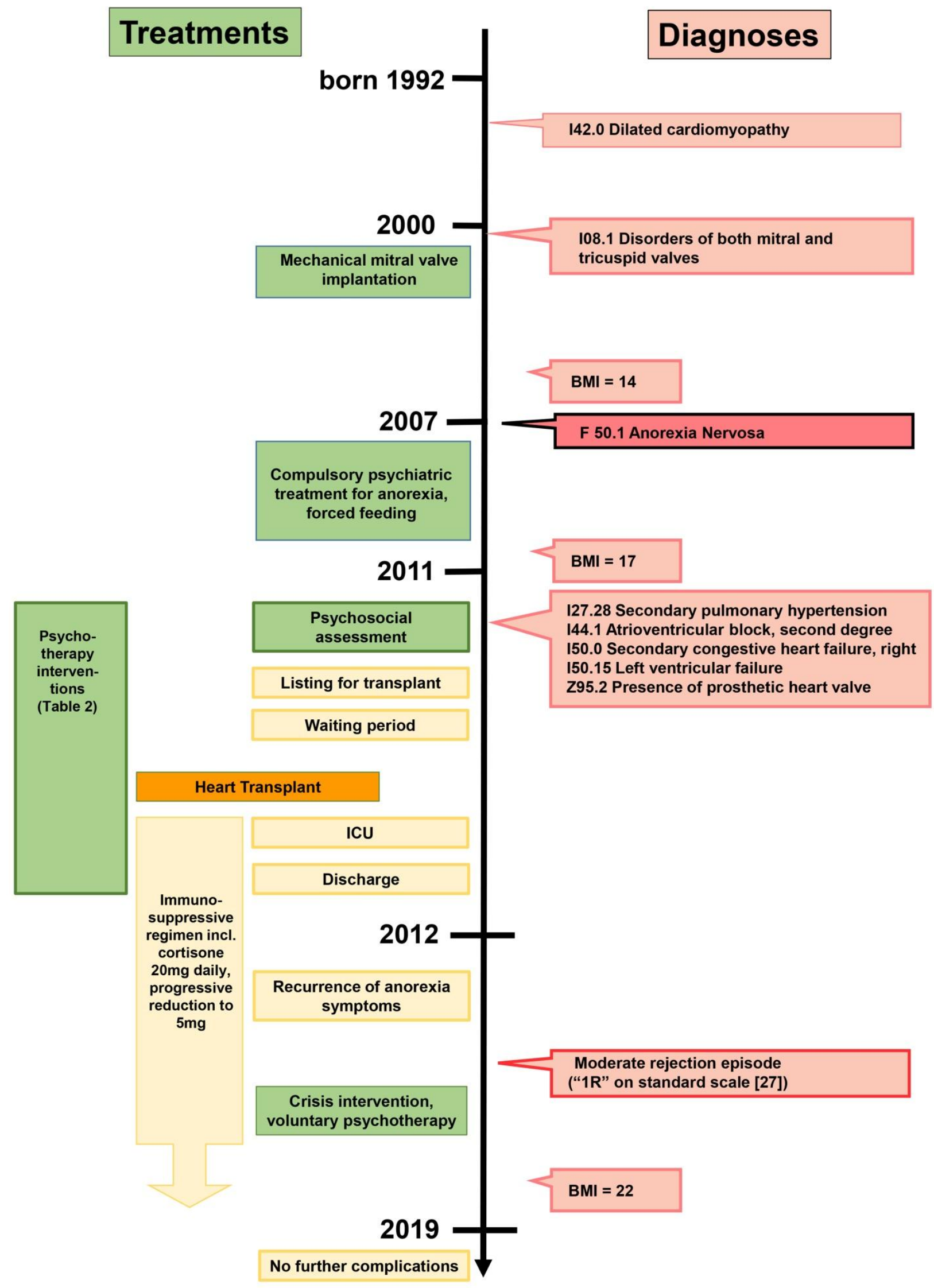

Figure 1 Timeline of diagnoses (ICD-10 [28]) and treatment. 
The two-fold challenge of having to deal with the trauma of a heart transplant and the psychiatric restrictions imposed by anorexia (pathological body image, negative controlling thoughts, setbacks in feelings of self-worth) was immense. Resource orientation, respect for autonomy, focus on the future, and hypnotherapeutic interventions all appear to have been helpful in this case. The psychotherapeutic process helped in the integration of the donor organ and interpretation of this donation as an enhancement of self-worth. For the attending psychotherapist it was important that the patient develop an internal detachment regarding her adolescent symptom-complex of eating, starving, and weight control with the goal of reducing vulnerability to manipulation. For this step, close therapeutic supervision was essential.

The rejection for treatment by another transplantation center turned out to be helpful. It was perceived as a warning by the patient and consequently spiked her motivation to seek an alternative center to get one "last chance." This should not be interpreted as a case of competition between heart transplant centers but of the utilization of diverging opinions about "transplantability" in order to encourage the recovery and maturity processes in an adolescent patient.

Our experience provides an additional illustration of how, as Skillings and Lewandowski (2015) argue, psychosocial support in multidisciplinary teams throughout the course of assessment and treatment enhance the quality of patient care in solid organ transplantation. In the case described here, the psychosocial support of the psychologist was central for two reasons. First, psychotherapeutic treatment utilized the patient's heightened motivation to activate hidden psychological resources and address the anorexia symptoms. The patient's boundaries had been violated early on by her mother, and because previous therapists attempted to control her as well, previous psychotherapy was unsuccessful. This episode of psychotherapy, in contrast, revolved around the mother-daughter conflict without violating the patient's boundaries. The use of hypnotherapy interventions proved to be very useful in avoiding reactance and clearing a path for the patient to accept her body with its new heart. Second, the psychotherapist's recommendations helped the team to try an innovative care approach that focused on heightening the patient's sense of autonomy. Teamwork ensured that the dieting and weighing process, which in this case was unusual, was handled by nurses as recommended by the psychotherapist and approved by the team. The nursing staff reported having been enriched by their role in this case, as they were involved to an unusually high degree in team discussions.

The application of lessons drawn from this case to other patients with anorexia is limited by the specific expression of the disorder in her case, especially the absence of vomiting and diarrhea, which increased the care team's confidence that immunosuppression would be absorbed. The psychotherapy resources of a high-volume transplantation center were also beneficial. Overall, anorexia should still remain a contraindication for heart transplantation, but the case demonstrates that successful heart transplantation is possible in patients with anorexia with careful patient assessment and intensive psychotherapeutic support in the context of multidisciplinary care.

\section{Conclusions}

A precondition for therapeutic adjustments for patients at higher risk of organ rejection due to mental health disabilities is the full integration of the mental health professional in the 
multidisciplinary transplantation care team. Medical professionals, especially surgeons, are trained to treat the body. In the past, some surgical teams may have tended to discount patients' mental health as a factor potentially influencing the outcome of surgery. However, the likelihood that patient mental health can affect transplant outcomes is underscored by the current evidence [4] and in some special cases like the one reviewed here it is possible and beneficial to adapt perioperative caretaking to mental health risks. Continuous cooperation with mental health professionals in care teams helps not only in the early identification of such cases but also in the development of effective, individualized, "unconventional" interventions for simultaneously addressing somatic and psychological needs in cases where mental health problems must be monitored and mitigated.

This case underscores the importance of additional research into the connections between psychotherapeutic interventions for psychological disorders and long-term health after solid organ transplant. In order to expand clinical knowledge of these patients, we recommend additional studies of interventions for persons with mental health issues and long-term health outcomes among transplant patients using multiple cases and a greater range of mental health disorders.

\section{Author Contributions}

All authors contributed original text or text revisions.

\section{Funding}

This research received no funding.

\section{Competing Interests}

The authors have declared that no competing interests exist.

\section{References}

1. Costanzo MR, Dipchand A, Starling R, Anderson A, Chan M, Desai S, et al. The International Society of Heart and Lung Transplantation Guidelines for the care of heart transplant recipients. J Heart Lung Transplant. 2010; 29: 914-956.

2. Roussel MG, Gorham N, Wilson L, Mangi AA. Improving recovery time following heart transplantation: the role of the multidisciplinary health care team. J Multidiscip Healthc. 2013; 6: 293-302.

3. Christopherson LK. Cardiac transplantation: A psychological perspective. Circulation. 1987; 75: 57-62.

4. Dew MA, DiMartini A, Dobbels F, Grady K, Jowsey-Gregoire S, al. e. The 2018 ISHLT/APM/AST/ICCAC/STSW recommendations for the psychosocial evaluation of adult cardiothoracic transplant candidates and candidates for long-term mechanical circulatory support. J Heart Lung Transplant. 2018; 37: 803-823.

5. OPTN-Organ Procurement and Transplantation Network. 2018. Bylaws Richmond, VA: https://optn.transplant.hrsa.gov/governance/bylaws/.

6. Deutscher Bundestag. 2017. [Revised Transplantation Law in the Version of the Bulletin of September 4, 2007 (BGBI. I S. 2206), Last Revised through Article 2 of the Law in the Version 
of July 18, 2017 (BGBI. I S. 2757)]. Berlin: Deutscher Bundestag.

7. Tigges-Limmer K, Brocks Y, Winkler Y, Stock Gissendanner S, Morshuis M, Gummert JF. Mental health interventions during ventricular assist device therapy: A scoping review. Interact Cardiov Thorac Surg. 2018; 27: 958-964.

8. Tigges-Limmer K, Rosendahl J, Strauss B, Albes JM, Dziewas R, Gummert J. [Psychological Interventions in Heart Surgery]. Z Herz- Thorax- Gefäßchir. 2011; 25: 29-37.

9. Heilmann C, Kuijpers N, Beyersdorf F, Berchtold-Herz M, Trummer G, Stroh AL, et al. Supportive psychotherapy for patients with heart transplantation or ventricular assist devices. Eur J Cardiothorac Surg. 2011; 39: e44-e50.

10. Dew MA, Kormos RL, DiMartini AF, Switzer GE, Schulberg HC, Roth LH, et al. Prevalence and risk of depression and anxiety-related disorders during the first three years after heart transplantation. Psychosomatics. 2001; 42: 300-313.

11. Jowsey SG, Taylor ML, Schneekloth TD, Clark MM. Psychosocial challenges in transplantation. J Psychiatr Pract. 2001; 7: 404-414.

12. Kuntz K, Weinland SR, Butt Z. Psychosocial challenges in solid organ transplantation. J Clin Psychol Med Settings. 2015; 22: 122-135.

13. Dew MA, DiMartini AF. Psychological disorders and distress after adult cardiothoracic transplantation. J Cardiovasc Nurs. 2005; 20: S51-S66.

14. Gagnier JJ, Kienle G, Altman DG, Moher D, Sox H, Riley D, et al. The CARE guidelines: Consensus-based clinical case reporting guideline development. Glob Adv Health Med. 2013; 2: 38-43.

15. Kobayashi S, Kai K, Okabe S, Tsutsui J, Tsutsumi T, Fuchinoue S, et al. New-onset anorexia nervosa after living kidney transplantation. Psychosomatics. 2018.

16. Berry $\mathrm{OO}$, Kymissis C. Key role of social supports in a cardiac transplant treatment team. J Psychiatr Pract. 2016; 22: 133-139.

17. Skillings JL, Lewandowski AN. Team-based biopsychosocial care in solid organ transplantation. J Clin Psychol Med Settings. 2015; 22: 113-121.

18. Cajita MI, Baumgartner E, Berben L, Denhaerynck K, Helmy R, Schonfeld S, et al. Heart transplant centers with multidisciplinary team show a higher level of chronic illness management - Findings from the International BRIGHT Study. Heart \& Lung: The Journal of Acute and Critical Care. 2017; 46: 351-356.

19. Aggarwal A, Kumar A, Gregory MP, Blair C, Pauwaa S, Tatooles AJ, et al. Nutrition assessment in advanced heart failure patients evaluated for ventricular assist devices or cardiac transplantation. Nutr Clin Pract. 2013; 28: 112-119.

20. Russo MJ, Iribarne A, Hong KN, Ramlawi B, Chen JM, Takayama H, et al. Factors associated with primary graft failure after heart transplantation. Transplantation. 2010; 90: 444-450.

21. Peura JL, Colvin-Adams M, Francis GS, Grady KL, Hoffman TM, Jessup M, et al. Recommendations for the use of mechanical circulatory support: device strategies and patient selection: A scientific statement from the American Heart Association. Circulation. 2012; 126: 2648-2667.

22. Mehra MR, Canter C, Hannan M, Semigran MJ, Uber P, Baran D, et al. The 2016 International Society for Heart Lung Transplantation listing criteria for heart transplantation: A 10-year update. J Heart Lung Transplant. 2016; 35: 1-23.

23. Burkhardt P. [The Therapeutic Tertium and Hypnotic Rituals]. In Revenstorf D, Burkhardt $P$ 
(Eds.) 2015. [Hypnosis in Psychotherapy, Psychosomatics und Medicine, 3rd Edition]. Berlin: Springer Verlag, pp. 49-51.

24. Trenkle B. [Utilization: A Core Concept of Ericksonian Hypnotherapie]. In Revenstorf D, Burkhardt P (Eds.) 2015. [Hypnosis in Psychotherapy, Psychosomatics und Medicine, 3rd Edition]. Berlin: Springer Verlag, pp. 83-88.

25. Seemann H. 2012. Assertiveness Training for Women, 4th Edition] Stuttgart: Klett-Cotta.

26. Tigges-Limmer K, Gummert J. [Hypnotherapeutic Interventions in Hearttransplantation]. Hypnose-Zeitschrift für Hypnose und Hypnotherapie 2010; 5: 18.

27. Stewart S, Winters GL, Fishbein MC, Tazelaar HD, Kobashigawa J, Abrams J, et al. Revision of the 1990 working formulation for the standardization of nomenclature in the diagnosis of heart rejection. J Heart Lung Transplant. 2005; 24: 1710-1720.

28. World Health Organization. International Statistical Classification of Diseases and Related Health Problems 10th Revision. Available online at https://icdwhoint/.

29. Collins TL, Wayne Holden E, Scheel JN. Cognitive functioning as a contraindication to organ transplant surgery: Dilemmas encountered in medical decision making. J Clin Psychol Med Settings. 1996; 3: 413-422.

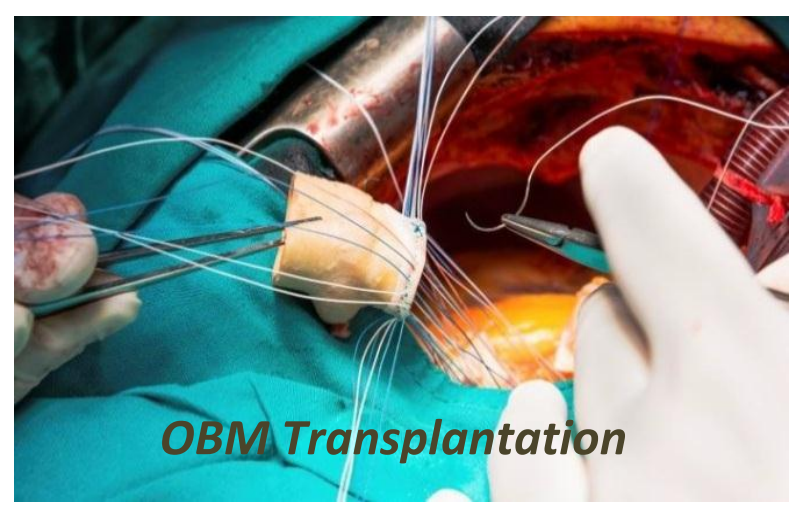

Enjoy OBM Transplantation by:

1. Submitting a manuscript

2. Joining in volunteer reviewer bank

3. Joining Editorial Board

4. Guest editing a special issue

For more details, please visit:

http://www.lidsen.com/journals/transplantation 